This item is the archived peer-reviewed author-version of:

\title{
Efficacy of different types of mobilization techniques in patients with primary adhesive capsulitis of the shoulder : a systematic review
}

\section{Reference:}

Noten Suzie, Meeus Mira, Stassijns Gaëtane, van Glabbeek Francis, Verborgt Olivier, Struyf Filip.- Efficacy of different types of mobilization techniques in patients with primary adhesive capsulitis of the shoulder : a systematic review

Archives of physical medicine and rehabilitation - ISSN 0003-9993 - 97:5(2016), p. 815-825

Full text (Publishers DOI): http://dx.doi.org/doi:10.1016/j.apmr.2015.07.025

To cite this reference: http://hdl.handle.net/10067/1328760151162165141 


\title{
Efficacy of Different Types of Mobilization Techniques in Patients With Primary Adhesive Capsulitis of the Shoulder: A Systematic Review
}

\author{
Suzie Noten, MSc, ${ }^{a, b}$ Mira Meeus, PhD, ${ }^{a, b, c}$ Gaetane Stassijns, MD, PhD, ${ }^{d}$ \\ Francis Van Glabbeek, MD, PhD, ${ }^{\text {e }}$ Olivier Verborgt, MD, PhD, ${ }^{\text {, }}$ f Filip Struyf, PhD $^{a, b}$
}

From the ${ }^{a}$ Department of Rehabilitation Sciences and Physiotherapy, Faculty of Medicine and Health Sciences, University of Antwerp, Antwerp; ${ }^{b}$ Pain in Motion Research Group, Belgium; ' ${ }^{C}$ Department of Rehabilitation Sciences and Physiotherapy, Faculty of Medicine and Health Sciences, Ghent University, Ghent; ${ }^{d}$ Department of Physical Medicine and Rehabilitation, Antwerp University Hospital, Edegem; ${ }^{e}$ Department of Orthopaedic Surgery and Traumatology, Antwerp University Hospital, Edegem; and ${ }^{f}$ Department of Orthopaedic Surgery and Traumatology, AZ Monica, Antwerp, Belgium.

\begin{abstract} capsulitis (AC) of the shoulder. identified by manual screening of the reference lists. 810 patients were included. on both pain and ROM.

Archives of Physical Medicine and Rehabilitation 2015;

Adhesive capsulitis (AC) of the shoulder is often defined as a disorder characterized by progressive pain and loss of active and passive mobility of the glenohumeral joint. The annual incidences are $3 \%$ to $5 \%$ in the general population and even up to $40 \%$ in people with diabetes. ${ }^{1,2}$ It mainly affects people between the ages
\end{abstract}

Objective: To systematically review the literature for efficacy of isolated articular mobilization techniques in patients with primary adhesive

Data Sources: PubMed and Web of Science were searched for relevant studies published before November 2014. Additional references were

Study Selection: All English language randomized controlled trials evaluating the efficacy of mobilization techniques on range of motion (ROM) and pain in adult patients with primary AC of the shoulder were included in this systematic review. Twelve randomized controlled trials involving

Data Extraction: Two reviewers independently screened the articles, scored methodologic quality, and extracted data for analysis. The review was conducted and reported according to the Preferred Reporting Items for Systematic Reviews and Meta-Analyses statement. All studies were assessed in duplicate for risk of bias using the Physiotherapy Evidence Database Scale for randomized controlled trials.

Data Synthesis: The efficacy of 7 different types of mobilization techniques was evaluated. Angular mobilization $(\mathrm{n}=2)$, Cyriax approach $(n=1)$, and Maitland technique $(n=6)$ showed improvement in pain score and ROM. With respect to translational mobilizations ( $=1)$, posterior glides are preferred to restore external rotation. Spine mobilizations combined with glenohumeral stretching and both angular and translational mobilization $(n=1)$ had a superior effect on active ROM compared with sham ultrasound. High-intensity mobilization $(n=1)$ showed less improvement in the Constant Murley Score than a neglect group. Finally, positive long-term effects of the Mulligan technique ( $\mathrm{n}=1$ ) were found

Conclusions: Overall, mobilization techniques have beneficial effects in patients with primary AC of the shoulder. Because of preliminary evidence for many mobilization techniques, the Maitland technique and combined mobilizations seem recommended at the moment.
Disclosures: none. of 40 and 60 years, with women more commonly affected than men. ${ }^{3}$ AC is mainly divided into 2 types in the literature, the idiopathic or primary form and the acquired or secondary form. Although no specific cause is identified in primary AC, the development of secondary AC is associated with recent surgery, immobilization, or trauma and also with systemic, extrinsic, or intrinsic disorders. Systemic disorders include a history of diabetes mellitus and thyroid disorders. ${ }^{4}$ Extrinsic disorders are not 
directly related to the shoulder and include cardiopulmonary diseases, cervical spine pathology, stroke, Parkinson disease, and humerus fractures. Intrinsic disorders are associated with the glenohumeral joint soft tissues or structures, including rotator cuff pathologies, biceps tendinitis, calcific tendinitis, and $\mathrm{AC}$ joint arthritis. ${ }^{1,5,6}$ AC lasts approximately 12 to 42 months in total and consists of 3 phases. It starts with a painful phase, which lasts 2 to 9 months. Subsequently, a stiff phase occurs (lasting 3-12mo), defined by stiffening and restriction of shoulder range of motion (ROM). The recovery phase is the final phase of the disease and is characterized by regaining movement and function over approximately 5 to 26 months. Some patients may not recover entirely and remain with some movement restriction. ${ }^{7}$ Additionally, after having $\mathrm{AC}$ on one side, the individual risk to develop $\mathrm{AC}$ in the contralateral shoulder increases by $5 \%$ to $34 \% .^{6}$

With AC, a decrease of capsular extensibility is seen as one of the most important pathologic mechanisms that results in large mobility deficits. Consequently, the restoration of glenohumeral motion is of great clinical importance to patients with AC because this would largely improve shoulder function. ${ }^{8,9}$

Kelley et $\mathrm{al}^{6}$ published current evidence-based recommendations and clinical practice guidelines for the treatment of patients with AC. The interventions were comprised of corticosteroid injections in the short term (4-6wk), patient education, physical modalities (ultrasound and electrical stimulation), joint mobilizations, translational mobilizations, manipulations, and stretching exercises. They concluded that some physiotherapeutic interventions show evidence regarding reduced pain or increased mobility in the short and long term.

As previously described, there are reasons to suggest that mobilization techniques may be effective in reducing pain and disability in patients with AC of the shoulder. Mobilization is defined as a low-velocity and small- or large-amplitude movement applied anywhere within a joint ROM $^{10}$ to improve the corresponding extensibility of the shoulder capsule and stretch the specific tightened soft tissues to induce beneficial effects. ${ }^{11}$ Mobilization techniques are commonly used to improve ROM and include both angular and translational mobilizations. Angular mobilizations are often applied as continuous passive motion or dynamic splinting. An external motorized device provides low-load continuous passive motion to move the joint passively through a specified ROM, creating a prolongedduration stretch. ${ }^{9}$ This is an established method of overcoming joint stiffness and histologically hypothesized for enhancing the healing of connective tissues. ${ }^{12,13}$ A shoulder splinting system was developed to apply a low-load prolonged-duration stretch to increase time at end range and achieve permanent elongation of connective tissue. ${ }^{14}$ By applying translational mobilizations, the humeral head is shifted in the preferred direction, while the elbow remains fixed. ${ }^{15}$ The therapist can either translate in an anterior, posterior, or inferior direction. ${ }^{16,17}$ In addition, individual mobilization techniques can be combined, which is implemented in for example Mulligan and Maitland techniques. The Mulligan technique ${ }^{18}$ includes a combination of sustained manual application of gliding force to the joint with a

\footnotetext{
List of abbreviations:

AC adhesive capsulitis

MeSH Medical Subjects Heading

PEDro Physiotherapy Evidence Database

ROM range of motion
}

simultaneous active movement of the joint by the patient. Studies that have used this technique on the elbow and ankle revealed a beneficial effect on pain and joint ROM. ${ }^{19,20}$ The Maitland technique $^{11}$ is based on the 5-grade classification system of Maitland and describes the amplitude of the rhythmic oscillating mobilization in the specified range of movement. Furthermore, mobilizations can be performed beyond the pain threshold. These so-called high-intensity techniques do not refer to the frequency that patients are treated, but they include active exercises up to and beyond the pain threshold, passive stretching, and manipulation of the glenohumeral joint and home exercises aimed at stretching and maximal reaching with the intent to restore ROM and reduce pain. ${ }^{21}$ Deep friction massage, as used by Cyriax and Russell, ${ }^{22}$ is often used before and in conjunction with mobilization techniques. The purpose of friction massage is to reduce abnormal fibrous adhesions and to make scar tissue more mobile in subacute and chronic inflammatory conditions by realigning the normative soft tissue fibers.

Many suggestions for mobilization techniques are available, but it is still a matter of debate what the optimal direction of force and movement application should be to restore joint mobilization in patients with $\mathrm{AC}$ of the shoulder. ${ }^{23}$ Therefore, it is of importance to compare the treatment effects of different mobilization techniques. The aim of this systematic review is to evaluate the efficacy of isolated articular mobilization techniques in patients with primary AC of the shoulder to identify which technique(s) may be most beneficial in the restoration of joint mobility and reduce pain in patients with $\mathrm{AC}$.

\section{Methods}

This systematic review is reported following the Preferred Reporting Items for Systematic reviews and Meta-Analyses guidelines. $^{24}$

\section{Eligibility criteria}

The Population, Intervention, Comparison, Outcome, and Study design method ${ }^{25}$ was used to derive keywords. The present systematic review attempted to include articles that described the results of clinical trials (S) evaluating the efficacy of isolated articular mobilization techniques (I) on ROM and pain (O) in patients with primary AC of the shoulder $(\mathrm{P})$. The comparison $(\mathrm{C})$ was undefined to evaluate the efficacy of any isolated mobilization techniques in patients with primary $\mathrm{AC}$ of the shoulder.

\section{Information sources and search strategy}

Both PubMed and Web of Science databases were searched to retrieve relevant articles. The search was conducted until November 2014. A prefabricated template was used for study selection designed by the Belgian Health Care Knowledge Centre. ${ }^{26}$ The following keywords were used frozen shoulder, adhesive capsulitis, periarthritis (Medical Subjects Heading [MeSH]), periarthritis, musculoskeletal manipulations (MeSH), musculoskeletal manipulations, manual therapy, manual techniques, manipulation, manual translation, articular translation, manual mobilization, manual mobilisation, mobilization, mobilisation, traction (MeSH), traction, glide, gliding, treatment outcome (MeSH), treatment outcome, therapy effect, efficacy, and effectiveness. 


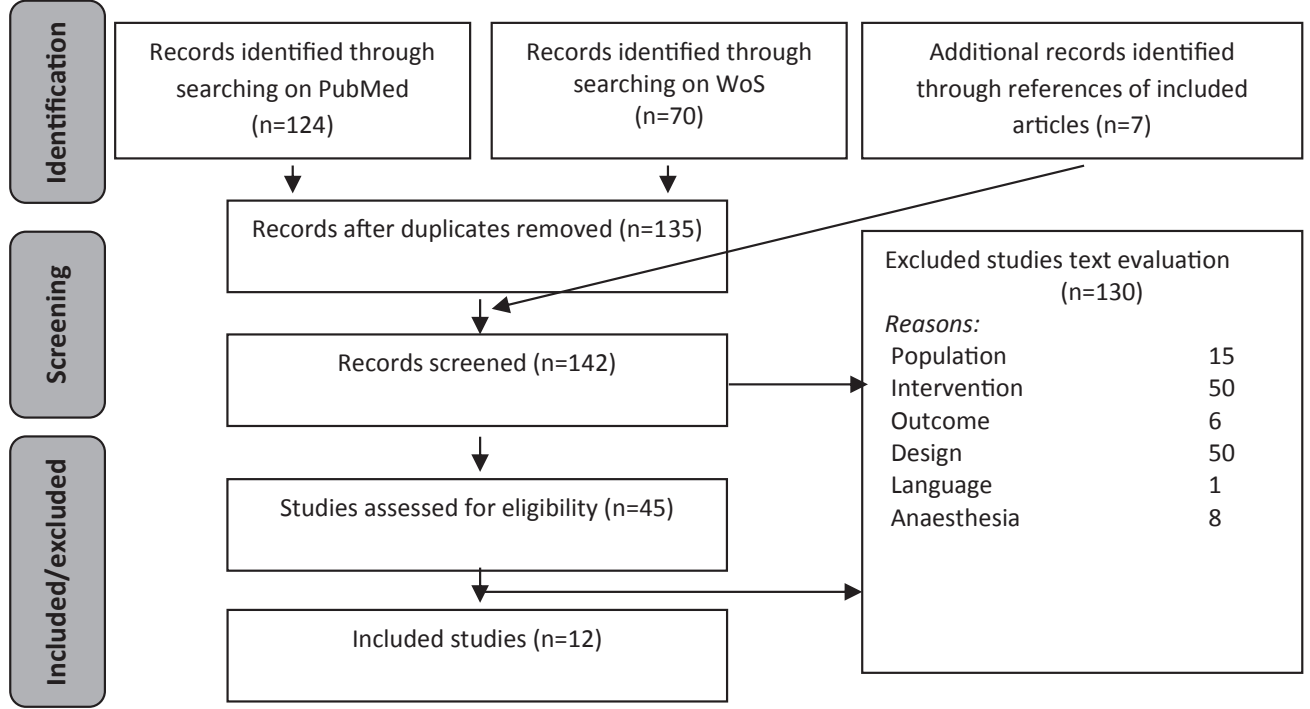

Fig 1 Flowchart of the study selection. Abbreviation: WoS, Web of Science.

\section{Study selection}

To be included in the present systematic review, articles had to meet the selection criteria noted in appendix 1.

\section{Data items and collection}

The following specific information was extracted from each included trial: (1) characteristics of the trial sample (number of participants, sex, age, stadium of the disease, and the trial's inclusion and exclusion criteria); (2) type of mobilization technique (mobilization modality, intervention frequency, and solely or combined with other treatment techniques); (3) type of control intervention; (4) outcome assessment; and (5) therapy effect (outcome measure, assessment intervals, and results). The included studies were divided between both review authors for data extraction and were checked by the other author. The methods of the included studies are heterogeneous (eg, length of follow-up and treatment period, sample differences); therefore, the approach of a box score or meta-analysis to quantify the results is not appropriate.

\section{Risk of bias in individual studies}

Methodologic quality was assessed independently by 2 researchers who were blinded from each other's quality assessment. After individually rating the selected articles, the ratings of both researchers were compared, and potential differences were discussed in a consensus meeting. Scorings were checked by a third researcher. Risk of bias in the different studies was assessed with the Physiotherapy Evidence Database (PEDro) Scale. ${ }^{27}$ According to the study design and risk of bias, studies could score a level of evidence A2 (randomized controlled trial of good quality, sufficient sample size, and double-blinded) or B (if previous criteria were not fulfilled). Recommendations are graded based on the level of evidence (www.cbo.nl).

\section{Results}

\section{Selection of studies}

The process of the study selection is presented in figure 1. Most studies were excluded based on the intervention. A total of 12 studies were included in the systematic review.

\section{Risk of bias and level of evidence}

As previously stated, all studies were evaluated with the PEDro Scale. There was a 98\% (130/133 items) agreement between the 2 researchers when scoring the selected items. After a second review, both researchers agreed on differences in rating. The final score of each study is presented in table 1. The methodologic quality varied between 4 and 10 out of 11 on the PEDro Scale. According to the PEDro classification, most of the studies showed a methodologic quality of level B. Many studies lost points on blinding of patients, ${ }^{8,9,21,23,28-34}$ therapist, $, 8,21,23,28-35$ and assessor. $^{9,21,23,28,30,34}$ Additionally, the concealment of allocation items was often not attained. ${ }^{8,9,21,28,30,31,34}$ Most studies scored well on randomization and comparability of groups. Only 1 study was double-blinded and received level of evidence A2. ${ }^{35}$

\section{Study characteristics}

To allow deeper interpretation and translation of the results, characteristics regarding the study population, intervention, follow-up period, and main results of the studies are presented in table 2. Level of conclusion of the most important outcome parameters is summarized in table 3 .

\section{Participants}

This review addressed 810 patients with primary AC with a mean age varying between $47.1^{34}$ and 58.9 years. ${ }^{28}$ Adult patients with 
Table 1 Results of the methodologic assessment of mobilization techniques in patients with primary AC

\begin{tabular}{|c|c|c|c|c|c|c|c|c|c|c|c|c|c|}
\hline \multirow[b]{2}{*}{ Author } & \multicolumn{11}{|c|}{ Criteria } & \multirow{2}{*}{$\begin{array}{l}\text { Quality } \\
\text { Score }\end{array}$} & \multirow{2}{*}{$\begin{array}{l}\text { Level of } \\
\text { Evidence }\end{array}$} \\
\hline & 1 & 2 & 3 & 4 & 5 & 6 & 7 & 8 & 9 & 10 & 11 & & \\
\hline Buchbinder et $\mathrm{al}^{35}$ & Yes & Yes & Yes & Yes & Yes & No & Yes & Yes & Yes & Yes & Yes & 10 & A2 \\
\hline Diercks and Stevens ${ }^{21}$ & Yes & Yes & No & Yes & No & No & No & Yes & Yes & Yes & Yes & 7 & B \\
\hline Doner et $\mathrm{al}^{28}$ & Yes & Yes & No & Yes & No & No & No & Yes & Yes & Yes & Yes & 7 & B \\
\hline Dundar et $\mathrm{al}^{9}$ & Yes & Yes & No & Yes & No & No & No & Yes & Yes & Yes & Yes & 7 & B \\
\hline Gaspar and Willis ${ }^{30}$ & No & No & No & Yes & No & No & No & No & Yes & Yes & Yes & 4 & B \\
\hline Guler-Uysal and Kozanoglu ${ }^{31}$ & Yes & Yes & No & Yes & No & No & Yes & Yes & Yes & Yes & Yes & 8 & B \\
\hline Johnson et $\mathrm{al}^{23}$ & Yes & Yes & Yes & Yes & No & No & No & Yes & Yes & Yes & Yes & 8 & B \\
\hline Kumar et $\mathrm{al}^{34}$ & Yes & Yes & No & Yes & No & No & No & Yes & Yes & Yes & Yes & 8 & B \\
\hline Paul et $\mathrm{al}^{33}$ & Yes & Yes & Yes & Yes & No & No & Yes & Yes & Yes & Yes & Yes & 9 & B \\
\hline Vermeulen et $\mathrm{al}^{8}$ & Yes & Yes & No & Yes & No & No & Yes & Yes & Yes & Yes & Yes & 8 & B \\
\hline Yang et $\mathrm{al}^{29}$ & Yes & Yes & Yes & Yes & No & No & Yes & No & Yes & Yes & Yes & 8 & B \\
\hline Yang et $\mathrm{al}^{32}$ & Yes & Yes & Yes & Yes & No & No & Yes & Yes & Yes & Yes & Yes & 9 & B \\
\hline
\end{tabular}

unilateral restricted shoulder movement ${ }^{33}$ or external rotation deficit $^{23,30}$ were included mostly if symptoms of pain and stiffness were present for a minimum of $2^{31,34}$ to 3 months. ${ }^{8,21,28,29,32,35}$ Most studies included patients in the stiff phase ${ }^{8,9,28-30,33} ; 2$ studies included both the painful and stiff phase, ${ }^{9,33}$ whereas the rest of the studies did not specifically define the phase. $^{21,23,31,32,34,35}$ Glenohumeral restrictions were further defined in a number of studies: 4 studies included patients with $50 \%$ loss of passive shoulder movement compared with the unaffected side, ${ }^{8,21,28,32} 1$ study reported a $25 \%$ loss of $\mathrm{ROM}^{29}$ and 1 study used a restriction of $30^{\circ}$ in 2 planes of movement. ${ }^{35}$ The aforementioned restrictions had to be present in at least $1^{8,28}$ or $2^{32,35}$ of the 3 movement directions (ie, forward flexion, abduction in the frontal plane, external rotation in $0^{\circ}$ or $90^{\circ}$ abduction). Corresponding exclusion criterion for patients was secondary AC of the shoulder, including rotator cuff pathologies, $9,29,31,32,35$ diabetes mellitus, ${ }^{21,29,32,34}$ a history of surgery on the affected shoulder, ${ }^{21,29,30,32,33}$ shoulder osteoarthritis, ${ }^{35}$ rheumatoid arthritis, $^{29,32}$ and neurologic disorders. ${ }^{8,23,34}$

\section{Type of mobilization techniques}

The following 7 types of mobilization techniques were evaluated: angular mobilization, ${ }^{9,30}$ translational mobilization, ${ }^{23}$ spine mobilizations combined with glenohumeral stretching and both angular and translational mobilization, ${ }^{35}$ high-intensity techniques beyond the pain threshold, ${ }^{21}$ Cyriax approach, ${ }^{31}$ Mulligan technique, ${ }^{28}$ and Maitland technique. ${ }^{8,29,30,32-34}$

\section{Outcome measures}

Most studies reported the effect of mobilization techniques on pain $^{8,9,23,28,31,33-35}$ and ROM..$^{8,9,23,28,30-35}$ Pain was measured using a visual analog scale $8,9,23,28,31,33,34$ or Likert Scale. ${ }^{35}$ In addition, the Constant Murley Score ${ }^{9,21}$ described pain and ROM after treatment.

\section{Study duration}

Frequency, total duration, and follow-up of all of the therapies are diverse. Frequency of therapies varied from $1^{35}$ to $5^{9,28,33,34}$ times a week. Total duration lasted 1 week ${ }^{31}$ up until 90 days. ${ }^{30}$ Followup fluctuated between 2 weeks $^{31}$ and 2 years. $^{21}$

\section{Effect of mobilization techniques}

It can be seen from the data in table 3 that 4 of 8 studies (all level B) reported reduced pain after a mobilization program. In addition, 8 of 10 (7 with level B, 1 with level A2) studies reported a beneficial effect of mobilization techniques on ROM.

\section{Effect of angular mobilization}

The utilized techniques regarding angular mobilizations were continuous passive motion ${ }^{9}$ and dynamic splinting. ${ }^{30}$ Dundar et $\mathrm{al}^{9}$ compared continuous passive motion with traditional therapy, consisting of pendulum exercises and stretching, and found a reduction in pain after continuous passive motion. No improvement in the Constant Murley Score (including pain and ROM evaluations) was found. Gaspar and Willis ${ }^{30}$ compared a cortical steroid injections with dynamic splinting, provided by the Dynasplint Shoulder System, the Maitland technique, ${ }^{11}$ and a combination of both. Dynamic splinting ${ }^{30}$ had a superior effect on ROM compared with the cortical steroid injections, but no significant difference between intervention groups was found.

\section{Effect of translational mobilization}

Johnson et $\mathrm{al}^{23}$ compared the effect of posterior and anterior glide mobilizations on ROM and pain. A reduction in pain was reported in both experimental groups, whereas the progression in ROM was favorable for posterior glide mobilizations. 
Table 2 Population characteristics, intervention, and results

\begin{tabular}{|c|c|c|c|c|c|c|}
\hline Author & Subjects & EI & CI & Assessment & Outcome & Results \\
\hline 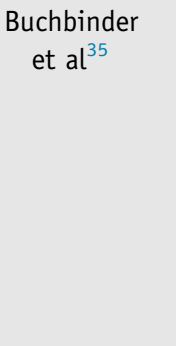 & $\begin{array}{l}\mathrm{N}=156 \\
\mathrm{~F}: 99 ; \mathrm{M}: 57 \\
55.0 \pm 9.3 \mathrm{y} \\
55.3 \pm 7.7 \mathrm{y} \\
\text { D0: } 12\end{array}$ & $\begin{array}{l}2 \text { times per wk, } 2 \text { wk to } 1 \text { time per } \\
\text { wk, } 4 \text { wk } \\
\text { Stretch muscles glenohumeral } \\
\text { joint } \\
\text { Cervicothoracic spine mobilization } \\
\text { Glenohumeral/p/accessory glide } \\
\text { and angular mobilization } \\
\text { Coordination and strength Rc and } \\
\text { scapular stabilizers }\end{array}$ & $\begin{array}{l}2 \text { times per wk, } 2 w k \text { to } 1 \text { time } \\
\text { per wk, } 4 w k \\
\text { Sham ultrasound }\end{array}$ & $\begin{array}{r}\text { Baseline, 6wk, } \\
12 w k, 26 w k\end{array}$ & $\begin{array}{l}\text { Pain (Likert Scale) } \\
\text { ROM/a/, FL, AB, ER, HBB }\end{array}$ & $\begin{array}{l}\mathrm{EI}=\mathrm{CI} \\
\uparrow \mathrm{EI}>\uparrow \mathrm{CI}\end{array}$ \\
\hline $\begin{array}{l}\text { Diercks and } \\
\text { Stevens }^{21}\end{array}$ & $\begin{array}{l}N=77 \\
F: 47 ; M: 30 \\
50 \pm 6 y \\
51 \pm 7 y\end{array}$ & $\begin{array}{l}\text { Physical therapy group }>\text { pain } \\
\text { threshold } \\
\text { Active exercises } \\
\text { Manipulation glenohumeral joint } \\
\text { Stretching and maximal reaching }\end{array}$ & $\begin{array}{l}\text { Supervised neglect group }<\text { pain } \\
\text { threshold } \\
\text { Pendulum exercises } \\
\text { Active exercises }\end{array}$ & $\begin{array}{c}1 \text { time per } 3 \mathrm{mo} \text {, } \\
\text { up to } 24 \mathrm{mo}\end{array}$ & Constant score & $\uparrow \mathrm{EI}<\uparrow \mathrm{CI}(3-18 \mathrm{mo})$ \\
\hline Doner et $\mathrm{al}^{28}$ & $\begin{array}{l}N=40 \\
F: 31 ; M: 9 \\
58.9 \pm 8.77 y\end{array}$ & $\begin{array}{l}5 \text { times per wk, } 3 w k \\
\text { Hot pack } \\
\text { TENS (20min, } 100 \mathrm{~Hz}, .05-.07 \mathrm{~ms} \text { ) } \\
\text { Mulligan technique (flexion, } \\
\quad \text { elevation, internal rotation) }\end{array}$ & $\begin{array}{l}5 \text { times per wk, } 3 \mathrm{wk} \\
\text { Hot pack } \\
\text { TENS }(20 \mathrm{~min}, 100 \mathrm{~Hz}, 0.05 \\
\quad-0.07 \mathrm{~ms}) \\
\text { Conventional passive } \\
\text { stretching }\end{array}$ & $\begin{array}{l}\text { Baseline, } 3 w k, \\
3 \text { mo }\end{array}$ & $\begin{array}{l}\text { Pain (VAS) } \\
\text { ROM } / \mathrm{a} /, / \mathrm{p} /, \mathrm{FL}, \mathrm{AB}, \mathrm{ER}, \\
\quad \mathrm{HBB}\end{array}$ & $\begin{array}{l}\uparrow E I>\uparrow C I \\
\uparrow E I>\uparrow C I\end{array}$ \\
\hline Dundar et $\mathrm{al}^{9}$ & $\begin{array}{l}N=57 \\
F: 39 ; M: 18 \\
56.3 \pm 7.8 y \\
57.1 \pm 8.3 y\end{array}$ & $\begin{array}{l}\text { 1h/d, } 5 \text { times per wk, } 4 \mathrm{wk} \\
\text { Continuous passive motion gradual } \\
\text { increase in motion } \\
\text { Home: Passive ROM, pendulum } \\
\text { exercises } \\
1 \text { time a d, } 12 \mathrm{wk}\end{array}$ & $\begin{array}{l}\text { 1h/d, } 5 \text { times per wk, } 4 \mathrm{wk} \\
\text { Conventional physiotherapy } \\
\text { treatment: active stretching, } \\
\text { pendulum exercises } \\
\text { Home: same }\end{array}$ & $\begin{array}{l}\text { Baseline, 4wk, } \\
\text { 12wk }\end{array}$ & $\begin{array}{l}\text { Pain (VAS) } \\
\text { ROM } \\
\text { Constant score }\end{array}$ & $\begin{array}{l}\uparrow E I>\uparrow C I \\
\uparrow E I=\uparrow C I \\
\uparrow E I=\uparrow C I\end{array}$ \\
\hline $\begin{array}{l}\text { Gaspar and } \\
\text { Willis }^{30}\end{array}$ & $\begin{array}{l}N=62 \\
55.6 \pm 7.9 y\end{array}$ & $\begin{array}{l}\text { Standard (EI1): } 2 \text { times per wk } \\
\text { Physical therapy } \\
\text { Therapeutic exercise } \\
\text { Moist heat } \\
\text { Education } \\
\text { Maitland end range } \\
\text { ROM/p/a/ } \\
\text { PNF; } \\
\text { Shoulder Dynasplint Systems (EI2): } \\
2 \text { times per d, 7d/wk } \\
\text { Combined (EI3): } \\
2 \text { times per wk } \\
\text { EI1 + EI2 }\end{array}$ & Cortical steroid injections & Baseline, 90d & $\mathrm{ROM} / \mathrm{a} /$, ER9O & $\begin{array}{l}\uparrow \mathrm{EI} 1=\mathrm{EI} 2=\mathrm{EI} 3 \\
>\uparrow \mathrm{CI}\end{array}$ \\
\hline
\end{tabular}




\begin{tabular}{|c|c|c|c|c|c|c|}
\hline Author & Subjects & EI & CI & Assessment & Outcome & Results \\
\hline $\begin{array}{l}\text { Guler-Uysal } \\
\text { and } \\
\text { Kozanoglu }\end{array}$ & $\begin{array}{l}N=40 \\
F: 28 ; M: 12 \\
56.0 \pm 8.6 y\end{array}$ & $\begin{array}{l}\text { CYR: } \\
\text { 1h, } 3 \text { times per wk, 1-2 w (>80\% } \\
\text { normative ROM) } \\
\text { CYR consisting of deep friction } \\
\text { massage and manipulation } \\
\text { Active stretching and pendulum } \\
\text { exercises } \\
\text { Home: passive ROM, pendulum } \\
\text { exercises } \\
1 \text { time a d }\end{array}$ & $\begin{array}{l}\text { PT: } \\
\text { 1h, } 5 \text { times per wk, 1-2wk } \\
\quad(>80 \% \text { normative ROM) } \\
\text { PT: } \\
\text { Hot pack (20min), } \\
\text { Short-wave diathermy (220V/ } \\
50 \mathrm{~Hz}, 20 \mathrm{~min}) \\
\text { Active stretching and } \\
\text { pendulum exercises } \\
\text { Home: same }\end{array}$ & $\begin{array}{l}\text { Baseline, 1wk, } \\
2 w k\end{array}$ & $\begin{array}{l}\text { Pain (VAS) } \\
\text { ROM } F L, A B, I R, E R\end{array}$ & $\begin{array}{c}\uparrow C Y R>\uparrow P T(N S) \\
\uparrow C Y R>\uparrow P T(2 w k) \\
\text { NOT ROM } \\
\text { AB }\end{array}$ \\
\hline $\begin{array}{l}\text { Johnson } \\
\text { et } \mathrm{al}^{23}\end{array}$ & $\begin{array}{l}N=18 \\
F: 14 ; M: 4 \\
54.7 \pm 8.0 y \\
50.4 \pm 6.9 y \\
D 0: 2\end{array}$ & $\begin{array}{l}\text { AM } \\
2-3 w k, 6 \text { sessions total } \\
\text { AM } \\
\text { Ultrasound }\left(1-3 \mathrm{MHz}, 1.5 \mathrm{~W} / \mathrm{cm}^{2} \text {, }\right. \\
\quad 10 \mathrm{~min} \text {, anterior) } \\
\text { Grade III mobilization } \\
\text { End-stretch position }>1 \mathrm{~min}, 15 \mathrm{~min} \\
\quad \text { total, } 6 \text { times }\end{array}$ & $\begin{array}{l}\text { PM } \\
2-3 w k, 6 \text { sessions total } \\
\text { PM } \\
\text { Ultrasound }(1-3 \mathrm{MHz}, 1.5 \mathrm{~W} / \\
\left.\mathrm{cm}^{2}, 10 \mathrm{~min}, \text { posterior }\right) \\
\text { Grade III mobilization } \\
\text { End-stretch position }>1 \mathrm{~min} \text {, } \\
\quad 15 \text { min total, } 6 \text { times }\end{array}$ & $\begin{array}{r}\text { Baseline, after } \\
\text { each session }\end{array}$ & $\begin{array}{l}\text { Pain (VAS) } \\
\text { ROM }_{E R}\end{array}$ & $\begin{array}{c}\uparrow A M=\uparrow P M \\
\uparrow A M<\uparrow P M \text { (session } \\
\text { 3-session 6) }\end{array}$ \\
\hline Kumar et $\mathrm{al}^{34}$ & $\begin{array}{l}N=40 \\
F: 14 ; M: 26 \\
47.9 y \\
47.1 y\end{array}$ & $\begin{array}{l}2-3 \text { glides } / \mathrm{s}, 30 \mathrm{~s}, 5 \text { sets, } 3 \text { times } \\
\text { per wk, } 4 \text { wk } \\
\text { CI plus Maitland mobilization } \\
\text { Glenohumeral caudal glides } \\
\text { Glenohumeral caudal glides } \\
\text { progression } \\
\text { Glenohumeral posteroanterior } \\
\text { glides } \\
\text { Passive oscillatory movements }\end{array}$ & $\begin{array}{l}10 \times 10 \text { s per exercise, } 5 \text { times } \\
\text { per wk, } 4 \text { wk } \\
\text { Supervised exercise program } \\
\text { Codman exercises } \\
\text { Shoulder wheel exercises } \\
\text { Wall-ladder exercises } \\
\text { Self-stretching exercises (AB, } \\
\quad \text { FL, ER, IR, AD) }\end{array}$ & Baseline, 4wk & $\begin{array}{l}\text { Pain (VAS) } \\
\text { ROM } E R, A B\end{array}$ & $\begin{array}{l}\uparrow E I>\uparrow C I \\
\uparrow E I>\uparrow C I\end{array}$ \\
\hline Paul et $\mathrm{al}^{33}$ & $\begin{array}{l}N=100 \\
F: 35 ; M: 65 \\
49.16 \pm 6.09 y \\
53.22 \pm 6.74 y\end{array}$ & $\begin{array}{l}\text { 20min, } 5 \text { times per wk, } 2 \mathrm{wk} \\
\text { CI plus weighted shoulder } \\
\text { countertraction during } \\
\text { mobilization, } 2-3 \mathrm{~kg} \\
\text { Glides in Maitland grades I-IV }\end{array}$ & $\begin{array}{l}20 \mathrm{~min}, 5 \text { times per wk, } 2 \mathrm{wk} \\
\text { Moist heat } \\
\text { Mobilization ( } 4 \text { sets, } 8-12 \\
\quad \text { times) } \\
\text { Home program } \mathrm{ROM} \text {, function } \\
\quad \text { exercises }(10 \times 3 / \mathrm{d})\end{array}$ & Baseline, 2wk & $\begin{array}{l}\text { Pain (VAS) } \\
\text { ROM }\end{array}$ & $\begin{array}{l}\uparrow E I=\uparrow C I \\
\uparrow E I=\uparrow C I\end{array}$ \\
\hline
\end{tabular}


Table 2 (continued)

\begin{tabular}{|c|c|c|c|c|c|c|}
\hline Author & Subjects & EI & CI & Assessment & Outcome & Results \\
\hline $\begin{array}{l}\text { Vermeulen } \\
\text { et } \mathrm{al}^{8}\end{array}$ & $\begin{array}{l}N=100 \\
F: 66 ; M: 34 \\
51.6 \pm 7.6 y \\
51.7 \pm 8.6 y \\
D 0: 4\end{array}$ & $\begin{array}{l}\text { HGMT: } \\
30 \mathrm{~min}, 2 \text { times per wk, up to } 12 \mathrm{wk} \\
\text { ( }>6 \mathrm{wk}+\mathrm{ROM}=\text { normal } \rightarrow 0-1 \text { time } \\
\text { per wk) } \\
\text { High-grade mobilization (Maitland } \\
\text { mobilization grades III and IV) } \\
\text { Inferior glides } \\
\text { Posterior and lateral glides } \\
\text { Anterior and medial glides } \\
\text { Oscillatory movements (caudal, } \\
\text { lateral and anterior) }\end{array}$ & $\begin{array}{l}\text { LGMT: } \\
\text { 30min, } 2 \text { times per wk, up to } \\
12 w k \\
\text { (>6wk+ROM=normal } \rightarrow 0-1 \\
\text { time per wk) } \\
\text { Low-grade mobilization } \\
\text { (Maitland mobilization grade } \\
\text { II) } \\
\text { Same glides and oscillatory } \\
\text { movements } \\
\text { 3min proprioceptive } \\
\text { neuromuscular facilitation/p/ } \\
2 \text { min Codman pendular } \\
\text { exercises } \\
\text { Without causing pain }\end{array}$ & $\begin{array}{c}\text { Baseline, } 3 \mathrm{mo}, \\
6 \mathrm{mo}, 12 \mathrm{mo}\end{array}$ & $\begin{array}{l}\text { Pain (VAS); ROM } \text { ER, } \\
/ \mathrm{a} /, / \mathrm{p} /\end{array}$ & $\begin{array}{c}\uparrow H G M T=\uparrow L G M T ; \\
\uparrow H G M T>\uparrow L G M T / \\
a / E R(12 \mathrm{mo}), / \mathrm{p} /, \text { ER, } \\
/ \mathrm{p} /, \text { AB (3 and 12mo) }\end{array}$ \\
\hline Yang et $\mathrm{al}^{29}$ & $\begin{array}{l}N=28 \\
F: 24 ; M: 4 \\
53.3 \pm 6.5 y \\
58 \pm 10.1 y \\
D 0: 7\end{array}$ & $\begin{array}{l}\text { A-B-A-C (EI1): } \\
2 \text { times per wk } 30 \text { min mobilization } \\
\quad \text { and simple exercises, } 12 \text { wk } \\
A=M R M, \text { Maitland } \\
B=E R M \\
C=M W M \\
10-15 \text { repetitions }\end{array}$ & $A-C-A-B(E I 2)$ & $\begin{array}{l}\text { Every } 3 w k \text { up to } \\
12 w k\end{array}$ & $\begin{array}{r}\text { FLEX-SF; FASTRAK } \\
\text { motion analysis }\end{array}$ & $\begin{array}{c}\uparrow E I 1=\uparrow E I 2 \text { for } \\
\text { ERM and MWM; } \\
\uparrow E R M=\uparrow M W M \\
\text { SHR: } \uparrow M W M>\uparrow E R M\end{array}$ \\
\hline Yang et $\mathrm{al}^{32}$ & $\begin{array}{l}N=32 \\
F: 22 ; M: 10 \\
54.3 \pm 7.6 y \\
56.8 \pm 7.2 y \\
54.9 \pm 10.3 y \\
D 0: 2\end{array}$ & $\begin{array}{l}\text { CrI: } \\
2 \text { times per wk, } 3 \text { mo } \\
\text { CC plus end-range mobilization } \\
\text { (Maitland grade IV) } \\
\text { Scapular mobilization } \\
\text { CC: } \\
2 \text { times per wk, 3mo } \\
\text { Midrange mobilization,/p/, } \\
\text { stretch, } \\
\text { physical modalities (ultrasound; } \\
\text { short-wave diathermy; } \\
\text { electrotherapy) } \\
\text { Active exercises }\end{array}$ & $\begin{array}{l}\text { Control: } \\
2 \text { times per wk, } 3 \text { mo } \\
\text { (larger shoulder kinematics } \\
\text { compared with CrI and CC) } \\
\text { CC }\end{array}$ & $4 w k, 8 w k$ & $\begin{array}{l}\text { FLEX-SF; FASTRAK } \\
\text { motion analysis } \\
\text { ROM/p/(hand behind } \\
\text { back; external } \\
\text { rotation; internal } \\
\text { rotation) }\end{array}$ & $\begin{array}{c}\uparrow C \mathrm{CI}>\uparrow C C(8 \mathrm{wk}) \\
\uparrow \mathrm{CrI}>\uparrow C C(8 \mathrm{wk}) ; \\
\uparrow C \mathrm{CI}>\uparrow C C(4 \\
-8 \mathrm{wk}) \\
\uparrow \mathrm{CrI}>\uparrow C C(8 \mathrm{wk}) \\
\uparrow \mathrm{CrI}>\uparrow C C \\
(4 \mathrm{wk}, 8 \mathrm{wk}) ; \uparrow \mathrm{CrI} \\
>\uparrow C C \\
(4 \mathrm{wk}, 8 \mathrm{wk}) ; \\
\uparrow \mathrm{CI}=\uparrow C C=\uparrow \mathrm{CrI}\end{array}$ \\
\hline
\end{tabular}

Abbreviations: /a/, active; $\mathrm{AB}$, abduction; $\mathrm{AD}$, adduction; $\mathrm{AM}$, anterior glide mobilization; $\mathrm{CC}$, criteria control; $\mathrm{CI}$, control intervention; $\mathrm{CrI}$, criteria intervention; $\mathrm{CYR}$, Cyriax; $\mathrm{DO}$, dropouts; $\mathrm{EI}$, experimental intervention; ER, external rotation; ERM, end-range mobilization; ER90, external rotation with the arm in $90^{\circ}$ of abduction; $F$, female; $F L$, flexion; FLEX-SF, flexion level scale of the shoulder function; HBB, hand behind back; HGMT, high-grade mobilization technique; IR, internal rotation; LGMT, low-grade mobilization technique; M, male; MRM, midrange mobilization; MWM, mobilization with movement; NS, not significant; /p/, passive; PM, posterior glide mobilization; PNF, proprioceptive neuromuscular facilitation; PT, physical therapy; RC, rotator cuff; SHR, scapulohumeral rhythm; TENS, transcutaneous electrical nerve stimulation; VAS, visual analog scale. 
Table 3 Level of conclusion of the most important results

\begin{tabular}{|c|c|c|c|c|}
\hline Outcome Variables & Studies & Type of Mobilization Techniques & $\begin{array}{l}\text { Level of } \\
\text { Evidence }\end{array}$ & $\begin{array}{l}\text { Level of } \\
\text { Conclusion }\end{array}$ \\
\hline \multirow[t]{8}{*}{ Pain } & + Dundar et $\mathrm{al}^{9}$ & Angular mobilization & B & \multirow[t]{8}{*}{ Moderate +} \\
\hline & + Guler-Uysal and Kozanoglu ${ }^{31}$ & Cyriax approach & B & \\
\hline & + Doner et $\mathrm{al}^{28}$ & Mulligan technique & B & \\
\hline & + Kumar et $\mathrm{al}^{34}$ & Maitland technique & B & \\
\hline & $=$ Johnson et $\mathrm{al}^{23}$ & Translational mobilization & B & \\
\hline & $=$ Paul et $\mathrm{al}^{33}$ & Maitland technique & B & \\
\hline & $=$ Vermeulen et $\mathrm{al}^{8}$ & Maitland technique & B & \\
\hline & $=$ Buchbinder et $\mathrm{al}^{35}$ & Combined technique & A2 & \\
\hline \multirow[t]{10}{*}{ ROM } & + Johnson et $\mathrm{al}^{23}$ & Translational mobilization & B & \multirow[t]{10}{*}{ Moderate +} \\
\hline & + Buchbinder et $\mathrm{al}^{35}$ & Combined techniques & $\mathrm{A} 2$ & \\
\hline & + Guler-Uysal and Kozanoglu ${ }^{31}$ & Cyriax approach & B & \\
\hline & + Doner et $\mathrm{al}^{28}$ & Mulligan technique & B & \\
\hline & + Gaspar and Willis ${ }^{30}$ & Angular and Maitland technique & B & \\
\hline & + Kumar et $\mathrm{al}^{34}$ & Maitland technique & B & \\
\hline & + Vermeulen et $\mathrm{al}^{8}$ & Maitland technique & B & \\
\hline & + Yang et $\mathrm{al}^{32}$ & Maitland technique & B & \\
\hline & $=$ Dundar et $\mathrm{al}^{9}$ & Angular mobilization & B & \\
\hline & $=$ Paul et $\mathrm{al}^{33}$ & Maitland technique & B & \\
\hline \multirow[t]{2}{*}{ Constant Murley Score } & $=$ Dundar et $\mathrm{al}^{9}$ & Angular mobilization & B & \multirow[t]{2}{*}{ Weak - } \\
\hline & - Diercks and Stevens ${ }^{21}$ & High-intensity mobilization & B & \\
\hline \multirow[t]{2}{*}{ FLEX-SF } & + Yang et $\mathrm{al}^{29}$ & Maitland technique & B & \multirow[t]{2}{*}{ Moderate +} \\
\hline & + Yang et $\mathrm{al}^{32}$ & Maitland technique & B & \\
\hline \multirow[t]{2}{*}{ FASTRAK } & + Yang et $\mathrm{al}^{29}$ & Maitland technique & B & \multirow[t]{2}{*}{ Moderate +} \\
\hline & + Yang et $\mathrm{al}^{32}$ & Maitland technique & B & \\
\hline
\end{tabular}

Abbreviations: FLEX-SF, flexion level scale of the shoulder function; + , positive result; - , negative result; $=$, equal result of mobilization techniques compared with conventional therapy.

\section{Effect of spine mobilizations combined with glenohumeral stretching and both angular and translational mobilization}

Buchbinder et $\mathrm{al}^{35}$ included spine mobilization, glenohumeral stretching, gliding, and angular mobilization in the experimental intervention and compared it with sham ultrasound. For active ROM, the combined technique proved to be superior, but no beneficial effects were found in terms of pain.

\section{Effect of high-intensity techniques beyond the pain threshold}

Diercks and Stevens ${ }^{21}$ included intensive mobilizations up to and beyond the pain threshold in addition to stretching and compared the results with a supervised neglect group receiving traditional therapy below the pain threshold. The Constant Murley Score was reported as an outcome variable, which showed less improvement with high-intensity techniques beyond the pain threshold.

\section{Effect of Cyriax approach}

Guler-Uysal and Kozanoglu ${ }^{31}$ compared a Cyriax approach of deep friction massage and mobilization exercises with a traditional therapy supplemented with a hot pack and short-wave diathermy. A positive effect of Cyriax on pain and ROM was reported.

\section{Effect of the Mulligan technique}

Doner et $\mathrm{al}^{28}$ compared the effect of the Mulligan technique with conventional stretching exercises. Both strategies were found to be effective in reducing pain and restoring ROM, but the immediate and long-term effects were in favor of the Mulligan technique.

\section{Effect of the Maitland technique}

Six studies made use of the Maitland technique as an intervention. ${ }^{8,29,30,32-34}$ As previously stated, Gaspar and Willis ${ }^{30}$ included this technique in their experiment; the effect on ROM was in favor of the intervention groups compared with cortical steroid injections. Paul et $\mathrm{al}^{33}$ found no superior effect of the Maitland technique on pain and ROM compared with mobilization in flexion and abduction stance. The Maitland technique had a beneficial effect on pain and ROM when compared with a supervised exercises program as used in the study of Kumar et al. ${ }^{34}$ A study by Vermeulen et $\mathrm{al}^{8}$ tried to unravel if there would be a difference between highgrade versus low-grade mobilization techniques, which resulted in a favorable effect of using high-grade mobilization on improving ROM. Two independent studies by Yang et al $^{29,32}$ implemented the Maitland technique, which showed significant progression on the flexion level scale of shoulder function in favor of end-range mobilization and mobilization with movement. In addition, both mobilizations showed improvement of the FASTRAK motion analysis outcomes. Hand behind back and external rotation ROM increased in the end range mobilization group compared with the midrange mobilization group. 


\section{Discussion}

\section{Summary of evidence}

Overall, mobilization techniques have beneficial effects in patients with primary AC of the shoulder, with strength of conclusions varying between moderate and preliminary evidence. Particularly, the Maitland technique and spine mobilizations combined with glenohumeral stretching and both angular and translational mobilization seem to be recommended at the moment. Because of the preliminary evidence, more studies are needed on assessing the effect of angular, translational, and high-intensity mobilization techniques; Cyriax approach; and Mulligan technique on pain and ROM.

The use of angular mobilization showed very limited preliminary evidence to reduce pain and improve ROM in primary AC (weak evidence) compared with corticosteroid injections or usual therapy. Angular mobilizations are preferable to corticosteroid injections, but no differences were found between intervention groups consisting of angular mobilization techniques, Maitland mobilizations, or a combination of both, ${ }^{30}$ which could be explained by a lack of power.

Preliminary evidence was found for the use of translational mobilization in primary AC. Only 1 study was found on the use of translational mobilization; therefore, the results must be interpreted with caution. Posterior glides proved to be superior to anterior glides to restore external rotation ROM, but optimal glide direction and duration of stretch mobilizations to restore ROM needs to be evaluated in further research. Care should be taken in generalizing the results of this study because of the small sample size and inclusion of only 1 therapist. $^{23}$

Preliminary evidence was also found for the effect of highintensity techniques beyond the pain threshold in patients with AC. According to their beliefs, Diercks and Stevens ${ }^{21}$ found an adverse effect of the high-intensity technique compared with the supervised neglect group on the Constant Murley Score. They suggested that intensive passive stretching may affect the natural course of the disease by activating the inflammatory reaction, when applied during the inflammation and proliferation stage and perhaps also during the early fibrotic stage. This indicates the importance of timing and therapy adjustments according to the different stages of AC. This study does not present detailed information about the composition of the techniques used.

Buchbinder $^{35}$ observed additional effects of spine mobilizations combined with glenohumeral stretching and both angular and translational mobilization on ROM for at least 6 months, which may be clinically important. The lack of pain reduction could be explained by the fact that there was less potential for additional effect of the device on this outcome. Further trials are needed to confirm the beneficial effects of the studied interventions and to determine whether other sequential treatments or a combination of treatments may result in better outcomes.

The Cyriax approach of deep friction massage and mobilization exercises showed very limited preliminary evidence on pain and ROM in the early phase of treatment. This technique is easily applicable because it does not require special equipment and anesthesia. However, long-term follow-up results are unknown and should be provided in future research. The exact mobilization exercises that were used in this study were not described properly.

Very limited preliminary evidence is found for the effect of the Mulligan technique on pain and ROM. The positive result of the Mulligan technique on pain and ROM should be interpreted with caution because it was only investigated in 1 study. This technique was chosen for the advantage of increasing ROM in addition to providing analgesia, but because it is a hands-on treatment, it is not possible to perform the study in a blinded manner. ${ }^{28}$

The Maitland technique showed a beneficial effect on ROM, flexion level scale of the shoulder function, and FASTRAK motion analysis outcomes. The study by Kumar ${ }^{34}$ showed that adding the Maitland technique to the supervised exercise program gives advantages in terms of pain and ROM. Mobilization techniques performed in the specific plane close to the end range improve the corresponding extensibility of the shoulder capsule and stretch the specific tightened soft tissues to induce beneficial effects. The neurophysiological effect could result from the rhythmic oscillatory movement of the Maitland technique that stimulates the peripheral mechanoreceptors and inhibits the nociceptive receptors. ${ }^{11}$ However, Paul ${ }^{33}$ did not find these superior effects on pain and ROM, which could be explained by the used measurement tool that may have been less reliable. Therefore, further studies, which establish the biomechanical rationale behind the effect of countertraction with appropriate tools, will need to be undertaken.

High-grade and low-grade mobilization in patients with primary AC yielded results according to expectations. Although the effect of the high-grade mobilization was superior, the low-grade group also achieved a considerable clinical improvement. Therefore, lowgrade mobilization could be the preferred treatment mode for those who are anxious about experiencing pain. The largest improvement was attained during the treatment itself, but ongoing progression of shoulder function was seen and can be explained by the initial improvement. ${ }^{8}$ Furthermore, because a control group was not included in this study, the findings may be the result of natural improvement. In addition, 2 other studies used this technique and found a beneficial effect of end-range mobilization and mobilization with movement in favor of the midrange mobilization techniques. ${ }^{29}$ This could be explained by the fact that the latter may only extend the adhesive capsule, whereas the end-range mobilization and mobilization with movement techniques can stretch the adhesive capsule and associated contracted periarticular structures. The appropriate treatment for each individual with primary AC of the shoulder may be dependent on the course and duration of symptoms. The multitreatment design limits the generalizability of the finding to normative clinical practice. Yang ${ }^{32}$ concluded that end-range mobilization and scapular mobilization are important techniques for primary $\mathrm{AC}$ of the shoulder. Subjects with larger shoulder kinematics were included in the control group. This homogenous subgroup was unlikely to improve with treatment, which could have biased the results.

\section{Study limitations}

This review has certain limitations that should be taken into account when interpreting its results. First, the main weakness of this review is the risk of bias; most studies failed to achieve blinding of the patients, $8,9,21,23,28-34$ therapist, $, 8,21,23,28-35$ and assessor, $9,21,23,28,30,34$ and concealment of allocation items $s^{8,9,21,28,30,31,34}$ were often not attained. Therefore, a note of caution is due here. However, only 1 of the 12 studies was not randomized, ${ }^{30}$ and in 1 study randomization was completed after patients had been allocated on the basis of shoulder kinematics. ${ }^{32}$

Second, characteristics of the included studies were heterogeneous. Inclusion criteria varied among most studies (eg, duration and classification of injury, magnitude of loss of ROM). Most of the 
mobilization techniques included patients in the stiff phase, whereas some studies did not specifically report the phase. It would seem reasonable that mobilization techniques would be most effective in the stiff phase to improve mobility, but not all studies took this into consideration. Therefore, the timing of the therapy at specific times in the disease's progress is an important issue for future research. In some studies the sample size was small, which may have resulted in a lack of statistical significance because of type II error (not enough power). ${ }^{8,9,23,28-32}$ Multiple treatment techniques and outcome measures were used, and the description of some utilized mobilization techniques was insufficient. For example, ROM was measured differently by most included studies, either active or passive ROM, total, or only glenohumeral $\mathrm{ROM},{ }^{36}$ and different positions were used (flexion, abduction, internal or external rotation, hand behind back). Therefore, the results must be interpreted with caution because marked heterogeneity was apparent for ROM. The use of ROM investigations should be normalized in further studies to generalize the results. It would not be ethical to use a sham group; therefore, the control group in most studies was also treated with therapy. In some studies hot packs were used to deliver superficial heating to increase the extensibility of collagen..$^{28,31,33}$ The application of heat has potentiated the effect of stretching on improving ROM in healthy people and may have influenced the results. ${ }^{37}$

Follow-up, total duration, and frequency of the therapy also varied among studies. Additionally, patient activity between posttest and follow-up were not always controlled. The benefits of the particular treatment over a longer follow-up period were unknown in most studies. As Struyf and Meeus ${ }^{36}$ previously mentioned, it is difficult to take the self-limiting aspect of AC into account. In most studies the follow-up period is limited to only 3 months, ${ }^{9,23,28-32,35}$ which seems to be insufficient knowing that AC can last up to several years. Although mobilization techniques seemed beneficial to reduce pain and increase ROM, there is little evidence to suggest that these techniques and physical therapy or other therapy modalities can alter disease prognosis and duration. ${ }^{6}$ Therefore, further research with a longer follow-up period is warranted to establish long-term effects.

\section{Conclusions}

Based on the present systematic literature review, overall mobilization techniques have beneficial effects in patients with primary AC of the shoulder. The Maitland technique and spine mobilizations combined with glenohumeral stretching and both angular and translational mobilizations seem to be recommended for the moment. Because of limited homogeneity and a limited number of studies with appropriate levels of evidence, more studies are needed on assessing the effect of angular, translational, and highintensity mobilization techniques; Cyriax approach; and Mulligan technique on pain and ROM.

\section{Keywords}

Bursitis; Rehabilitation; Review [publication type]

\section{Corresponding author}

Filip Struyf, PhD, Universiteit Antwerpen, Department of Rehabilitation Sciences and Physiotherapy, Universiteitsplein 1, 2610 Antwerpen. E-mail address: filip.struyf@uantwerpen.be.
Appendix 1 Study selection criteria

\begin{tabular}{|c|c|}
\hline Inclusion Criteria & Exclusion Criteria \\
\hline $\begin{array}{l}\text { - Adult patients with primary AC } \\
\text { of the shoulder, in any stadium } \\
\text { - The study assessed the efficacy } \\
\text { of all kinds of articular } \\
\text { mobilization techniques } \\
\text { - The outcome measure is pain } \\
\text { or ROM to assess the efficacy } \\
\text { of the treatment } \\
\text { - Clinical trials published in full } \\
\text { text } \\
\text { - Studies in English or Dutch } \\
\text { - Full-text available }\end{array}$ & $\begin{array}{l}\text { - Secondary AC of the shoulder } \\
\text { - Manipulations under anes- } \\
\text { thesia of the affected shoulder } \\
\text { - Case reports, reviews, letters } \\
\text { to the editor, clinical trials, } \\
\text { trial of an intervention, and } \\
\text { retrospective studies }\end{array}$ \\
\hline
\end{tabular}

\section{References}

1. Tasto JP, Elias DW. Adhesive capsulitis. Sports Med Arthrosc 2007; 15:216-21.

2. Huang YP, Fann CY, Chiu YH, et al. Association of diabetes mellitus with the risk of developing adhesive capsulitis of the shoulder: a longitudinal population-based followup study. Arthritis Care Res (Hoboken) 2013;65:1197-202.

3. Hand C, Clipsham K, Rees JL, Carr AJ. Long-term outcome of frozen shoulder. J Shoulder Elbow Surg 2008;17:231-6.

4. Cakir M, Samanci N, Balci N, Balci MK. Musculoskeletal manifestations in patients with thyroid disease. Clin Endocrinol (Oxf) 2003; 59:162-7.

5. Zuckerman JD, Rokito A. Frozen shoulder: a consensus definition. J Shoulder Elbow Surg 2011;20:322-5.

6. Kelley MJ, Shaffer MA, Kuhn JE, et al. Shoulder pain and mobility deficits: adhesive capsulitis. J Orthop Sports Phys Ther 2013;43: A1-31.

7. Lubiecki M, Carr A. Frozen shoulder: past, present, and future. J Orthop Surg (Hong Kong) 2007;15:1-3.

8. Vermeulen HM, Rozing PM, Obermann WR, le Cessie S, Vliet Vlieland TP. Comparison of high-grade and low-grade mobilization techniques in the management of adhesive capsulitis of the shoulder: randomized controlled trial. Phys Ther 2006;86: 355-68.

9. Dundar U, Toktas H, Cakir T, Evcik D, Kavuncu V. Continuous passive motion provides good pain control in patients with adhesive capsulitis. Int J Rehabil Res 2009;32:193-8.

10. Maitland G, Hengeveld E. Maitland's vertebral manipulation. 7th ed. London: Elsevier; 2005.

11. Maitland G. Peripheral Manipulation. 2nd ed. London: Butterworths; 1977.

12. Laupattarakasem W. Short term continuous passive motion. A feasibility study. J Hand Surg [Br] 1988;70:802-6.

13. Ada L, Goddard E, McCully J, Stavrinos T, Bampton J. Thirty minutes of positioning reduces the development of shoulder external rotation contracture after stroke: a randomized controlled trial. Arch Phys Med Rehabil 2005;86:230-4.

14. Hepburn G. Contracture and stiff joint management with Dynasplint. J Orthop Sports Phys Ther 1987;8:498-504.

15. Edmond S. Manipulation and mobilization: extremities and spinal techniques. St Louis: Mosby; 1993.

16. Kaltenborn F. Manual therapy of the extremity joints. Oslo: Olaf Norlis, Bokhandel; 1973.

17. Roubal PJ, Dobritt D, Placzek JD. Glenohumeral gliding manipulation following interscalene brachial plexus block in patients with adhesive capsulitis. J Orthop Sports Phys Ther 1996;24:66-77.

18. Mulligan B. "Manual Therapy "“NAGS", SNAGS", "MWMS" etc." Wellington: Pl view Serv. Ltd; 1999. 
19. O'Brien T, Vicenzino B. A study of the effects of Mulligan's mobilization with movement treatment of lateral ankle pain using a case study design. Man Ther 1998;3:78-84.

20. Abbott JH. Mobilization with movement applied to the elbow affects shoulder range of movement in subjects with lateral epicondylalgia. Man Ther 2001;6:170-7.

21. Diercks RL, Stevens M. Gentle thawing of the frozen shoulder: a prospective study of supervised neglect versus intensive physical therapy in seventy-seven patients with frozen shoulder syndrome followed up for two years. J Shoulder Elbow Surg 2004;13:499-502.

22. Cyriax J, Russell G. Textbook of Orthopaedic Medicine. 10th ed. London: Bailliere, Tindall \& Cassell Ltd; 1980.

23. Johnson AJ, Godges JJ, Zimmerman GJ, Ounanian LL. The effect of anterior versus posterior glide joint mobilization on external rotation range of motion in patients with shoulder adhesive capsulitis. J Orthop Sports Phys Ther 2007;37:88-99.

24. Moher D, Liberati A, Tetzlaff J, Altman DG. PRISMA Group. Preferred reporting items for systematic reviews and meta-analyses: the PRISMA statement. Int J Surg 2010;8:336-41.

25. Stone PW. Popping the (PICO) question in research and evidencebased practice. Appl Nurs Res 2002;15:197-8.

26. Thiry. KCE - Belgian Health Care Knowledge Centre. Template Study Sel., 2010.

27. Maher CG, Sherrington C, Herbert RD, Moseley AM, Elkins M. Reliability of the PEDro scale for rating quality of randomized controlled trials. Phys Ther 2003;83:713-21.

28. Doner G, Guven Z, Atalay A, Celiker R. Evalution of Mulligan's technique for adhesive capsulitis of the shoulder. J Rehabil Med 2013; 45:87-91.
29. Yang JL, Chang CW, Chen SY, Wang SF, Lin JJ. Mobilization techniques in subjects with frozen shoulder syndrome: randomized multiple-treatment trial. Phys Ther 2007;87:1307-15.

30. Gaspar PD, Willis FB. Adhesive capsulitis and dynamic splinting: a controlled, cohort study. BMC Musculoskelet Disord 2009; 10:111.

31. Guler-Uysal F, Kozanoglu E. Comparison of the early response to two methods of rehabilitation in adhesive capsulitis. Swiss Med Wkly 2004; 134:353-8.

32. Yang JL, Jan MH, Chang CW, Lin JJ. Effectiveness of the end-range mobilization and scapular mobilization approach in a subgroup of subjects with frozen shoulder syndrome: a randomized control trial. Man Ther 2012;17:47-52.

33. Paul A, Rajkumar JS, Peter S, Lambert L. Effectiveness of sustained stretching of the inferior capsule in the management of a frozen shoulder. Clin Orthop Relat Res 2014;472:2262-8.

34. Kumar A, Kumar S, Aggarwal A, Kumar R, Das PR. Effectiveness of Maitland techniques in idiopathic shoulder adhesive capsulitis. ISRN Rehabil 2012;2012:1-8.

35. Buchbinder R, Youd JM, Green S, et al. Efficacy and costeffectiveness of physiotherapy following glenohumeral joint distension for adhesive capsulitis: a randomized trial. Arthritis Rheum 2007; 57:1027-37.

36. Struyf F, Meeus M. Current evidence on physical therapy in patients with adhesive capsulitis: what are we missing? Clin Rheumatol 2014; 33:593-600.

37. Nakano J, Yamabayashi C, Scott A, Reid WD. The effect of heat applied with stretch to increase range of motion: a systematic review. Phys Ther Sport 2012;13:180-8. 